\title{
SPECTRAL AND ELECTRICAL DIAGNOSTICS OF GLIDING ARC
}

\author{
A. Czernichowsiri, H. Nassar, A. Ranaivosoloarimanana \\ GREMI, University of Orléans, 45067 Orléans cedex 2, France
}

\author{
A.A. Fridman
}

Kurchatov Institute, 123182 Moscow, Russia

M. SIMEK

Institute of Plasma Physics, 18211 Prague, Czech Republic

K. Musiol, E. Pawelec

Jagiellonian University, Reymonta 4, 30-059 Cracow, Poland

AND L. DitTrichova

Technical University, 61669 Brno, Czech Republic

(Received January 9, 1996)

Using spectroscopic and electric measurements, vibrational and rotational molecular gas temperatures as well as free electron temperature and concentration were determined in different regions of a time-periodical type, atmospheric pressure non-equilibrium low current gliding arc. It was shown that this discharge includes an initial quasi-equilibrium zone, with the quasi-equilibrium temperature of 4 to $6 \mathrm{kK}$, and the non-equilibrium zone with the electron temperature about $10 \mathrm{kK}$, the vibrational temperature about $3 \mathrm{kK}$, rotational and translational temperatures from 1 to $1.5 \mathrm{kK}$. The transition between two mentioned zones coincides with the phenomenon of the arc "length explosion" already observed in moderate-current gliding arc.

PACS numbers: 33.10.Jz, 52.70.Kz, 52.25.Kn

\section{Introduction}

In the previous papers $[1,2]$ we described some properties of the so-called gliding arc (or GlidArc), a quasi periodic electrical discharge, which has been used for several applications in chemistry and environment protection [3]. This powerful (up to $40 \mathrm{~kW}$ ) discharge can be easily obtained between at least two divergent electrodes, with a gas flowing between them at pressures ranging from 
soft vacuum to several atmospheres. It was also explained how this discharge can produce a relatively cold non-equilibrium plasma with a complicated time-space structure including quasi-equilibrium (hot) and non-equilibrium (cold) periods, as well as a Fast Equilibrium to Non-Equilibrium TRansition (which we call FENETRe) between them $[4,5]$. During the FENETRe an ionization instability creates conditions for existence of a relatively cold plasma and at this moment, the phenomenon of the arc "length explosion" takes place. More detailed theory about the FENETRe is in preparation.

Previous spectral measurements of such gliding discharges already indicated striking differences between rotational, vibrational and electron temperatures.

A low DC-current ( 0.15 to $0.25 \mathrm{~A}$ ) GlidArc, burning in slightly hydrogenated argon, was studied by Sember and Svoboda [6]. In their experiment the mean excitation temperature was measured from the Boltzmann plot of ArI lines and the electron density $N_{\mathrm{e}}$ was determined from the hydrogen $\mathrm{I}_{\beta}$ line broadening. In the discharge initiation point, these quantities were equal to $4.3 \pm 0.4 \mathrm{kK}$ and $3 \times 10^{14} \mathrm{~cm}^{-3}$, respectively. In the developed discharge zone the temperature and $N_{\mathrm{e}}$ values decreased to $3.6 \pm 0.2 \mathrm{kK}$ and $2 \times 10^{14} \mathrm{~cm}^{-3}$, respectively. The electron concentrations were therefore much higher than expected for the plasma in the local thermodynamic equilibrium (LTE) state at the measured temperatures. The $N_{\mathrm{e}}$ values, derived form the $\mathrm{H}_{\beta}$ broadening, corresponded rather to the temperature of $7 \mathrm{kK}$ (in the LTE state). When more hydrogen was added to the discharge $(2 \%)$, the electron density in the initiation and main part of the GlidArc increased to $1 \times 10^{15}$ and $5 \times 10^{14} \mathrm{~cm}^{-3}$ respectively, while the excitation temperatures changed only a little.

Another diagnostics of the gliding AC-discharge burning in air, was recently presented by Tesar et al. [7]. For the average arc current of $0.1 \mathrm{~A}$, authors described the following discharge areas between horn-shaped electrodes:

- a disruptive spark discharge (SD) zone in the narrowest part of electrodes separation and closely above it $(\approx 30 \mathrm{~mm}$ in the gas flow $=$ direction $)$, with a high electric field strength and current density in the discharge channel,

- a disintegrating corona and spark discharges (DD), expanded by the blowing stream of air, having the length of about $70 \mathrm{~mm}$.

All temperatures in the SD area, determined from the $\mathrm{N}_{2}$ and $\mathrm{OH}$ molecular emission spectra, were about $500 \mathrm{~K}$ higher than temperatures in the DD zone. For example, in the SD zone the vibrational temperature $T_{\mathrm{v}}$ determined from the population of the upper $C^{3} \Pi_{\mathrm{u}}$ state of $\mathrm{N}_{2}$, was equal to $3740 \mathrm{~K}$, while the rotational temperature $T_{\mathrm{r}}$ determined from the same $\mathrm{N}_{2}$ band was equal to $2600 \mathrm{~K}$. The corresponding values for the DD zone were equal to 3200 and $2060 \mathrm{~K}$, respectively.

In the present paper, the non-equilibrium properties of the low-current arc will be proved by spectroscopic measurements of the $\mathrm{N}_{2}$ second positive system and electrophysical observations.

\section{Experimental measurements and results}

The gliding arc device is schematically shown in Fig. 1. Two diverging blade-shaped steel electrodes are placed in a relatively fast air flow $(>10 \mathrm{~m} / \mathrm{s})$ as 


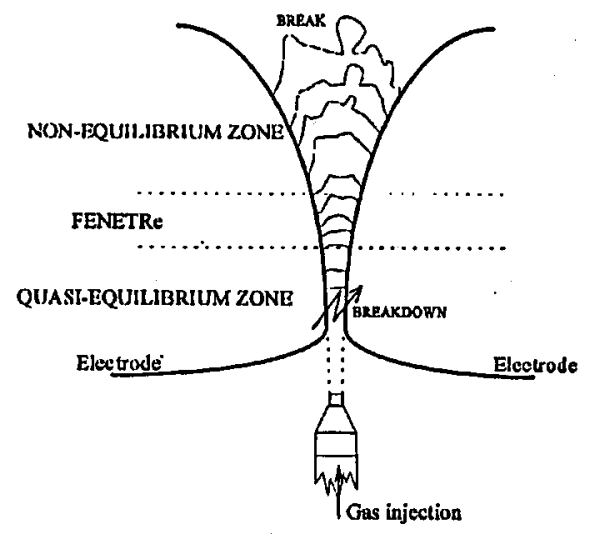

Fig. 1. Gliding arc electrical scheme.

indicated in the figure. The electrodes are connected to a commercial high-inductance neon-transformer (50 Iz, $10 \mathrm{kV}$ at open circuit, $0.13 \mathrm{~A}$ at the short circuit).

The spark discharge starts at the place of the shortest distance (1-2 mm) between two electrodes. During the time of about $1 \mu \mathrm{s}$ a low resistance plasma channel is formed which causes a fall of the voltage between electrodes. This small plasma filament, in the state close to the LTE, is then dragged up by the gas flow and the arc column length increases together with the voltage. The arc current is almost constant during this quasi-equilibrium period but the arc voltage is increasing. The total arc power increases also almost linearly with the discharge length. This evolution is terminated, when the arc length approaches to a critical value, for which the growing plasma resistance becomes equal to the external one, and the maximum value of discharge power corresponds to the half of generator maximal power. At the same time, the voltage, the electric field and power approach their maximum values. When the moving arc length exceeds the critical value, the arc heat losses continue to increase. But, since the electrical power cannot increase any more, it is no longer possible to sustain the arc in the quasi-thermodynamic equilibrium state. The gas temperature falls rapidly and the plasma conductivity is maintained by the high value of electron temperature and stepwise ionization.

After this fast transition (FENETRe, see Fig. 1), the gliding arc continues its evolution but in non-equilibrium state. The specific heat losses in this regime become much smaller than in equilibrium one. Therefore, the discharge length can increase up to other critical value, when the arc breaks and a new discharge starts again from the initial breakdown. The final breaking point is defined by the geometry of electrodes, by the flow conditions and characteristics of the power supply. In the case of an $\mathrm{AC}$ high-inductance power supply, one can observe a phenomenon of the multiple zero-current crossings, without the discharge interruption, during several periods of the discharge evolution.

About $80 \%$ of the total gliding arc power is dissipated after the FENETRe, during the non-equilibrium period. It means that gliding arc may really be useful as an efficient and very simple generator of the non-equilibrium plasma $[4,5]$. 
To show directly the non-equilibrium nature of the gliding arc burning in air at atmospheric pressure, the vibrational $\left(T_{v}\right)$ and rotational $\left(T_{r}\right)$ temperatures were measured in the initial (quasi-equilibrium) and final (post-FENETRe) discharge regions. The rotational temperature is supposed to be close to the kinetic gas temperature $T_{0}$. The electron temperature $T_{\mathrm{e}}$ and free electron concentration $N_{\mathrm{e}}$ were evaluated from electric parameters and other discharge characteristics for both, the initial and final discharge regions (see below). The "length explosion" phenomenon during transition stage (FENETRe) has been already demonstrated [5] by high speed camera pictures of another medium-current gliding arc burning in air.

\subsection{Vibrational and rotational temperatures}

Because of quasi-periodic nature of the gliding arc (with 5 to $30 \mathrm{~ms}$ as a quasi-period) the time integrated emission spectroscopy was used to obtain a sufficiently high signal. Light was collected by two lenses, imaged onto the input slit $(0.15 \mathrm{~mm})$ of the $1 \mathrm{~m} \mathrm{McPherson}$ monochromator. The monochromator was equipped with the $1200 \mathrm{G} / \mathrm{mm}$ ruled grating, blazed at $300 \mathrm{~nm}$ and an UV sensitive photomultiplier as the light detector. The photomultiplier signal was averaged by the PAR boxcar averager. The boxcar output signal was stored in a 4-channel digital oscilloscope.

Two observation areas were selected: the quasi-equilibrium "before the FENETRe" zone and non-equilibrium "after the FENETRe". An analysis of time integrated air emission spectra shows intense molecular bands in the region from $200 \mathrm{~nm}$ to $400 \mathrm{~nm}$ (with maximum around $350 \mathrm{~nm}$ ). Band shapes and their relative intensities depend strongly on the observation position. The $\mathrm{N}_{2}, \mathrm{~N}_{2}^{+}, \mathrm{NO}$ and $\mathrm{OH}$ bands were detected, the last one was due to an ambient air humidity which was used as plasma-forming gas. Some of bands had quite low intensities in the "after FENETRe" region, and were merged in the signal noise or even disappeared (e.g. the $\mathrm{N}_{2}$ band). The spectra were registered during a slow scanning $(1 \mathrm{~nm} / \mathrm{min})$ of the monochromator.

In order to trigger the boxcar, while the arc filament image was passing the monochromator input slit, the light reflected from the input slit blades was collected by a quartz fiber and detected by a second photomultiplier. Output from the second photomultiplier was used to trigger the boxcar at the moment of maximum light intensity. The boxcar gate width ranged from 10 to $100 \mu \mathrm{s}$ depending on the length of the light pulses.

Bands of the 2 nd positive system of nitrogen molecule $C^{3} \Pi_{\mathrm{u}}-B^{3} \Pi_{\mathrm{g}}$ in $\Delta v=$ $\pm 2, \pm 1$, and 0 sequences were studied in different positions along the axis of the discharge. The monochromator spectral band pass $(0.1$ to $0.2 \mathrm{~nm})$ was low enough to recover partially resolved structure of the emission band for determination of vibrational and rotational temperatures.

Spectra of the 2 nd positive system were analyzed by the numerical procedure searching for the best $T_{\mathrm{v}}$ and $T_{\mathrm{r}}$ temperature values to fit theoretically calculated spectrum to the experimental band shape. The monochromator instrumental function was measured by means of a low-pressure mercury lamp spectrum. 
Two different fitting procedures were used: first one (marked as $A$ ) was elaborated in the Institute of Plasma Physics, Czech Academy of Sciences, and second one $(B)$ which originates from the University of Orléans. No correction for the spectral sensitivity of the optical system has been done. It was not necessary because of the proper combination of the photomultiplier type and grating efficiency, this spectral sensitivity may be considered as constant for analysis of the applied rotational spectrum.

Using the $A$ procedure $[8,9]$ the rotational temperatures were found by fitting the calculated spectrum to the beginning of $\Delta v= \pm 2, \pm 1$ or 0 sequences, after the numerical smoothing of data and background subtraction. The calculation model was based on high-resolution analysis of the rotational system by Roux et al. $[10,11]$ and Hönl-London factors [12]. The $(0,2),(0,1)$ and $(0,0) N_{2}$ bands were used for $T_{\mathrm{r}}$ determination in the "before FENETRe" region (almost the same $T_{\mathrm{r}}$ were obtained from all bands), whereas only $(0,1)$ band could be analyzed for the "post-FENETRe" region. For vibrational temperatures, the data evaluation technique consisted of the band-profiles generation for given $T_{\mathbf{r}}$ and instrumental function, with subsequent subtraction from experimental spectra in order to find the best fit. Resulting relative populations of the $C^{3} \Pi_{\mathrm{u}}$ state in two vibronic levels of $v=0$ and $v=1$ determined the $T_{\mathrm{v}}$ value.

Using the $B$ procedure [13-16], the rotational and vibrational temperatures were found by unique fit over a large complex spectrum of the overlapping $\mathrm{N}_{2}$ bands covering $\Delta v= \pm 2, \pm 1$ and 0 sequences, without: preliminary numerical smoothing, baseline subtraction and assumption that apparatus function is known. The band simulation was based on the same method as in procedure $A$. Generally, our program permits to simulate a molecular band without limits in wave numbers $v^{\prime}$ and $v^{\prime \prime}$, but in the case of the $C^{3} \Pi_{\mathrm{u}}-B^{3} \Pi_{\mathrm{g}}$ transitions and in order to gain a time, we have simulated only the overlapped $(0,0),(1,1),(2,2),(3,3),(4,4),(0,1)$, $(1,2),(1,0),(2,1),(0,2),(1,3),(2,4)$ and $(3,5)$ bands between the selected signal wave numbers. To determine the $T_{\mathrm{r}}$ and $T_{\mathrm{v}}$ from a given measured noisy spectrum, the simulated spectrum was compared point-by-point using a standard best-fit criterion. Four parameters were fitted: $T_{\mathrm{r}}, T_{\mathrm{v}}$, the apparatus width (supposed to be Gaussian) and background level.

To test the noise sensitivity of the determined temperature, we have simulated noisy "experimental" spectra: for the apparatus profile corresponding to the Gaussian width of $\Delta X=7 \mathrm{~cm}^{-1}$, the noise-to-signal ratio of $0.025,0.05$ and 0.1 and $T_{\mathrm{r}}=T_{\mathrm{v}}=3 \mathrm{kK}$. The applied best-fit criterion was found to be the good one, since the relative error of the derived temperatures had the same order of magnitude as the noise-to-signal ratio in the applied simulated "experimental" spectra: $\Delta T / T=0.015,0.025$ or 0.04 , respectively.

Figure 2 presents four measured and four corresponding calculated spectra (procedure $B$ ) for two characteristic regions of the gliding arc. The vibrational and rotational temperatures, found in initial equilibrium and final non-equilibrium zones of the gliding arc are given in Table. The experimental results obtained from the first fitting procedure are marked as $A$, those derived from the second procedure are marked as $B$. From Table one can see that in the initial quasi-equilibrium zone, the vibrational and rotational temperatures are almost the same and quite high, 


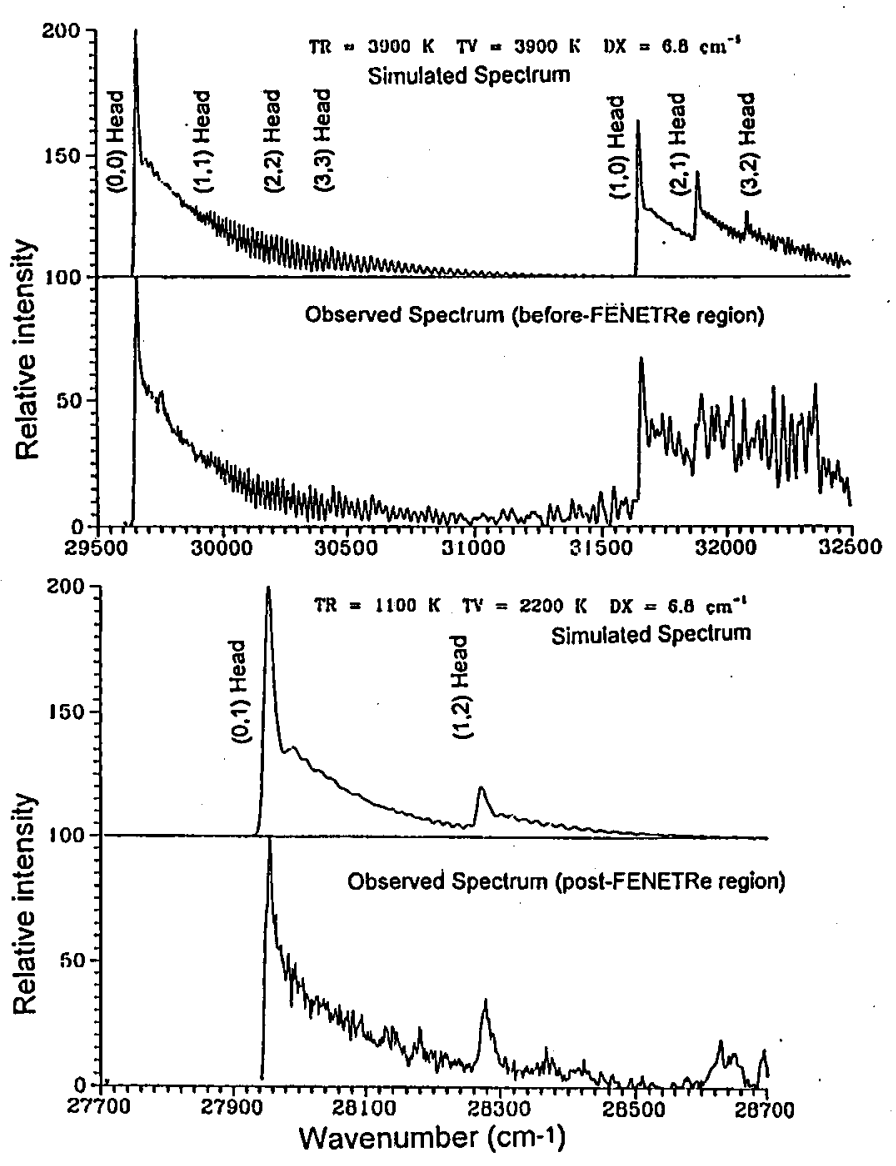

Fig. 2. An example of the simulated and observed spectra both for before and post-FENETRe zones in two different ranges of wave numbers.

TABLE

Experimental characteristics of the gliding arc equilibrium and non-equilibrium zones.

\begin{tabular}{c|c|c|c|c}
\hline \hline Zone & $T_{\mathrm{e}}$ & $T_{\mathrm{v}}$ & $T_{\mathrm{r}}=T_{0}$ & $N_{\mathrm{e}}$ \\
\hline \multicolumn{5}{c}{ Quasi-equilibrium } \\
\hline Model $A$ & $6 \mathrm{kK}$ & $3300 \pm 200 \mathrm{~K}$ & $2600 \pm 300 \mathrm{~K}$ & $10^{13} \mathrm{~cm}^{-3}$ \\
Model $B$ & $6 \mathrm{kK}$ & $3900 \pm 100 \mathrm{~K}$ & $3900 \pm 100 \mathrm{~K}$ & $10^{13} \mathrm{~cm}^{-3}$ \\
\hline \multicolumn{5}{c}{ Non-equilibrium } \\
\hline Model $A$ & $10 \mathrm{kK}$ & $2600 \pm 300 \mathrm{~K}$ & $1700 \pm 400 \mathrm{~K}$ & $10^{12} \mathrm{~cm}^{-3}$ \\
Model $B$ & $10 \mathrm{kK}$ & $2200 \pm 200 \mathrm{~K}$ & $1100 \pm 300 \mathrm{~K}$ & $10^{12} \mathrm{~cm}^{-3}$
\end{tabular}


about $4 \mathrm{kK}$. In the final non-equilibrium zone, the vibrational temperature remains on the high level of about $3 \mathrm{kK}$, but the rotational temperature falls to a relatively low level of $1-1.5 \mathrm{kK}$.

\subsection{Electron temperature and concentration}

Free electron parameters were determined for the same regions of the gliding arc where spectroscopic measurements were done. These parameters were estimated from measurements of instantaneous values of the discharge voltage and current.

Electrical signals corresponding to arc voltage and current were registered and stored by a four-channel digital oscilloscope. Signals from the photomultiplier, used for described above plasma spectroscopy, were collected simultaneously by the same oscilloscope, in order to have temperatures, voltage and current measured at the same moment. Values of the near electrodes voltage drop were measured separately. It is interesting that the voltage drop in the initial, "before FENETRe" region, is usually lower than that in the "after FENETRe" region. It corresponds to the non-equilibrium nature of the second region. Spatial characteristics of the discharge, lengths and cross-sections, were estimated also at the same moments and places of the gliding arc as the spectroscopic and electrophysical measurements.

The electric field $E$ was determined from the measured values of discharge voltages and lengths. The reduced electric field $E / n=E k T_{0} / p$ was then calculated from known pressure $p$ and translational temperature $T_{0}=T_{\mathrm{r}}$ (obtained from spectroscopic experiments). The electron temperature was then determined from its known dependence on the $E / n$ ratio in the air $[17,18]$. Corresponding results are given in Table.

The electron concentration $N_{\mathrm{e}}$ was derived from discharge current density and electron mobility (which is estimated also as known function of the specific electric field $E / n$ in air) $[17,18]$. Corresponding results are also presented in Table.

One can see from Table that in the quasi-equilibrium zone of the gliding arc the electron temperature is not much different from other temperatures, and the electron concentration is relatively high. Our results are therefore in complete accordance with the theoretical model considered in Refs. [4] and [5]. In the non-equilibrium zone, the electron temperature $T_{\mathrm{e}} \approx 10 \mathrm{kK}$ is much higher than the vibrational one $T_{v} \approx 3 \mathrm{kK}$, which in turn is higher than rotational and translational ones: $T_{\mathbf{r}} \approx T_{0} \approx 1$ to $1.5 \mathrm{kK}$. Such a hierarchy of temperatures, as well as relatively low electron concentration, with respect to the equilibrium plasma, are in good agreement with the theory of the non-equilibrium plasma $[17,18]$. On the other hand, such plasma parameters are very typical of powerful non-equilibrium discharges like a microwa ve plasma [18].

\section{Conclusions}

The complicated time-space structure of the gliding arc was experimentally investigated. It was shown by spectroscopic and electrophysical measurements that the initial quasi-equilibrium ("before FENETRe") plasma of the gliding arc has a temperature of the order $4-6 \mathrm{kK}$. The share of dissipated electrical power in this 
zone can be about $20-25 \%$ of the total power, which is a relatively small part of the total discharge energy.

It was proved experimentally that "post-FENETRe" plasma has strong non-equilibrium parameters. According to the spectroscopic and electrophysical diagnostics, the electron temperature was there about $10 \mathrm{kK}$, the molecular gas vibrational temperature was about $3 \mathrm{kK}$ and was consequently much higher than the rotational and translational ones $(1$ to $1.5 \mathrm{kK})$. Similar results were shown independently in Refs. [6] and [7].

Electron concentration after the FENETRe phase is about $10^{12} \mathrm{~cm}^{-3}$, smaller than in the initial zone, but the main part of the gliding arc power (up to $80 \%$ ) could be dissipated just in this non-equilibrium zone.

The transition between two mentioned discharge zones includes the phenomenon called the gliding arc "length explosion". This spectacular phenomenon illustrates the FENETRe phase itself and was experimentally observed with a high speed camera $[5,19,20]$.

The gliding arc, the continuous powerful atmospheric pressure non-equilibrium discharge, can be successfully applied for different chemical reaction [3] with the high energy efficiency and selectivity as well as for example non-equilibrium microwave discharge. It can be illustrated already by high efficiency of $\mathrm{CO}_{2}$ dissociation, $\mathrm{H}_{2} \mathrm{~S}$ decomposition, and sulfur reduction from $\mathrm{SO}_{2}$ in gliding arc.

Moreover, the moderate gas temperature $\left(T_{0}=1-1.5 \mathrm{kK}\right)$ permits to perform in the gliding arc chemical reactions with complicated kinetics (for example such as $\mathrm{N}_{2} \mathrm{O}$ oxidation to $\mathrm{NO}+\mathrm{NO}_{2}$, or dehalogenation of concentrated organic), which could not be effectively stimulated in the room temperature non-equilibrium plasma.

\section{References}

[1] H. Lesueur, A. Czernichowski, J. Chapelle, Coll. Phys., Suppl.au J. Phys. 51, C5-49 (1990).

[2] H. Lesueur, A. Czernichowski, J. Chapelle, Coll. Phys., Suppl. au J. Phys. 51, C5-57 (1990).

[3] A. Czernichowski, Pure Appl. Chem. 66, 1301 (1994).

[4] V.D. Rusanov, A.S. Petrusev, B.V. Potapkin, A.A. Fridman, A. Czernichowski, J. Chapelle, Sov. Phys.-Doklady 332, 306 (1993).

[5] A.A. Fridman, A. Petrousov, J. Chapelle, J.M. Cormier, A. Czernichowski, H. Lesueur, J. Stevefelt, J. Phys. III (France) 4, 1449 (1994).

[6] V. Sember, P. Svoboda, in: Proc. 16th Symp. on Plasma Physics and Technology, Prague 1993, p. 230.

[7] C. Tesar, J. Janca, V. Kapicka, in: Proc. XIth Int. Symp. on Physics of Switching Arc, Brno 1994, p. 74.

[8] M. Simek, Acta Phys. Slov. 42, 83 (1992).

[9] S. DeBenedictis, G. Dilecce, M. Simek, Acta Phys. Univ. Comeniae 33, 159 (1992).

[10] F. Roux, F. Michaud, M. Vervloet, J. Mol. Spectrosc. 67, 143 (1989).

[11] F. Roux, F. Michaud, M. Vervloet, J. Mol. Spectrosc. 158, 270 (1993).

[12] I. Kovacs, Astrophys. J. 145, 643 (1966). 
[13] A. Czernichowski, J. Phys. D 20, 559 (1987).

[14] H. Nassar, A. Czernichowski, J. Phys. (France) 51, C5-289 (1990).

[15] H. Nassar, A. Czernichowski, Acta Phys. Pol. A 84, 215 (1993).

[16] H. Nassar, A. Czernichowski, in: Proc 11th Int. Symp. on Plasma Chemistry, ISPC.11, Loughborough (England) 1993, Vol. 1, p. 487.

[17] Y.P. Raizer, The Physical Fundamentals of Gas Discharge Processes, Nauka, Moskva 1980 (in Russian).

[18] V.D. Rusanov, A.A. Fridman, Physics of Chemically Active Plasma, Nauka, Moskva 1984 (in Russian).

[19] J.M. Cormier, F. Richard, J. Chapelle, M. Dudemaine, in: Proc. 2nd Int. Conf. on Electr. Contacts, Arcs, Apparatus and their Applications, Xi'an (China) 1993, p. 40.

[20] J.M. Cormier, F. Richard, J. Chapelle, in Proc. 7th Int. Conf. on Switching Arcs Phenomena, Eódź (Poland) 1993, p. 313. 